\title{
Airway issues in anaplastic thyroid carcinoma
}

\author{
Ashok R. Shaha • Alfio Ferlito $\cdot$ Randall P. Owen $\cdot$ Carl E. Silver • \\ Juan P. Rodrigo $\cdot$ Missak Haigentz Jr $\cdot$ William M. Mendenhall • \\ Alessandra Rinaldo $\cdot$ Robert C. Smallridge
}

Received: 3 April 2013/Accepted: 3 May 2013/Published online: 21 May 2013

(C) Springer-Verlag Berlin Heidelberg 2013

\section{Introduction}

Anaplastic thyroid carcinoma is the least common but most lethal of thyroid cancers $[1,2]$. It is distressing that even after half a century of active investigation we have not made substantial progress in the management of this unique neoplasm. In 1983, Kim and Leeper [3] showed promising results with the addition of doxorubicin as a chemotherapeutic agent. These results, however, have not been duplicated [4]. Whether this is related to the many stages of differentiation of anaplastic thyroid carcinoma, or the inability to separate poorly differentiated and anaplastic thyroid carcinoma, remains unclear at this time. The classic

This paper was written by members and invitees of the International Head and Neck Scientific Group (http://www.IHNSG.com).

\footnotetext{
A. R. Shaha

Head and Neck Service, Memorial Sloan-Kettering Cancer

Center, New York, NY, USA
}

\section{A. Ferlito $(\square) \cdot$ A. Rinaldo}

ENT Clinic, University of Udine, Piazzale S. Maria della

Misericordia, I-33100 Udine, Italy

e-mail: a.ferlito@uniud.it

\section{R. P. Owen}

Division of Metabolic, Endocrine and Minimally Invasive Surgery, Department of Surgery, Mount Sinai School of Medicine, New York, NY, USA

\section{E. Silver}

Departments of Surgery and Otolaryngology-Head and Neck Surgery, Albert Einstein College of Medicine, Montefiore Medical Center, Bronx, NY, USA

\section{J. P. Rodrigo}

Department of Otolaryngology, Hospital Universitario Central de Asturias, Oviedo, Spain giant and spindle cell anaplastic thyroid carcinoma grows rapidly and the average survival in this group of patients is between 3 and 6 months. Fortunately it appears that there is a decreasing incidence of true anaplastic thyroid carcinoma at least in the United States (1-2\%). However, in areas with endemic goiter or neglected thyroid cancer, anaplastic thyroid carcinoma is still prevalent, forming 2-3\% of all thyroid cancers [5]. A tertiary cancer center generally has large numbers of patients seen on a referral basis and thus has the greatest experience with such a lethal and rare disease. However, even in those institutions where a larger number of anaplastic thyroid carcinomas are seen, we have not achieved major progress in the control or survival rate of this disease. Recently, there seems to be considerable interest in the development of multidisciplinary protocols to treat patients with anaplastic thyroid carcinoma by

J. P. Rodrigo

Instituto Universitario de Oncología del Principado de Asturias, Oviedo, Spain

M. Haigentz Jr

Division of Oncology, Department of Medicine, Albert Einstein College of Medicine, Montefiore Medical Center, Bronx, NY, USA

W. M. Mendenhall

Department of Radiation Oncology, University of Florida,

Gainesville, FL, USA

R. C. Smallridge

Division of Endocrinology, Department of Internal Medicine, Mayo Clinic, Jacksonville, FL, USA 
aggressively combining surgical and non-surgical modalities. Unfortunately, the number of patients who can undergo R0 resection is very small, and surgeons continue to find it difficult to perform a gross total resection and to maintain an adequate airway.

Thyroid cancer is a spectrum of diseases. On one end of the spectrum there is papillary thyroid carcinoma, one of the more indolent malignancies with a survival rate of more than $98 \%$. At the other end of the spectrum is anaplastic thyroid carcinoma, one of the most dreaded cancers, with a mortality rate of $98 \%$. The reason for the differences in biological behavior remains unclear. Whether there is a genetic progression from one end of the spectrum to the other remains to be seen. Molecular testing shows distinct genetic aberrations in anaplastic thyroid carcinoma. In the spectrum of aggressiveness of thyroid cancer, there appears to be a progressive sequence from well-differentiated thyroid carcinoma, to tall cell, to insular, to poorly differentiated, and lastly to anaplastic thyroid carcinoma.

One of the most common and difficult problems in the management of anaplastic thyroid carcinoma is securing the airway. The purpose of this editorial is to define some of these issues and propose practical considerations for management of the airway. Almost every patient with anaplastic thyroid carcinoma faces the problem of securing and maintaining a safe airway, and a large number of patients succumb eventually to airway obstruction. The American Thyroid Association (ATA) Anaplastic Thyroid Cancer Guidelines Taskforce deliberated over the last 3 years to develop a series of recommendations for the evaluation and management of anaplastic thyroid carcinoma [6]. This is a very important document which should be read by all physicians who treat patients with anaplastic thyroid carcinoma. The airway issues are discussed in R25$\mathrm{R} 28$. We will elaborate on some of these issues in this manuscript.

\section{Clinical presentation and diagnosis}

The majority of patients present with a rapidly increasing mass in the thyroid region of the neck. Quite often, patients will give a history of a thyroid mass for a long period of time and even a previous benign needle biopsy result, followed by transformation and rapid growth of anaplastic thyroid carcinoma. The majority of patients will present with discomfort in the neck, a rapidly growing mass, occasional shortness of breath, dysphagia, weight loss, dyspnea and orthopnea. Rarely, the patient may present with hemoptysis indicating extension of the tumor into the trachea. An occasional patient may present initially with distant metastasis, although the great majority present with a rapidly growing neck mass. It is vitally important to make an accurate diagnosis of these tumors. Fine needle aspiration biopsy will often direct the physician to consider anaplastic thyroid carcinoma. Definitive diagnosis can be obtained with a core biopsy. Core biopsy can be easily performed under local anesthesia during an office visit. Satisfactory samples of tissue should be sent for pathological and molecular investigations.

It is extremely important to distinguish poorly differentiated thyroid carcinoma from anaplastic thyroid carcinoma. Poorly differentiated thyroid carcinoma, a separate entity, may be misdiagnosed in many institutions as anaplastic thyroid carcinoma giving rise to an inaccurate record of satisfactory long-term survival. It is also vitally important to rule out lymphoma. Lymphoma of the thyroid gland may present as a bulky, poorly differentiated mass, difficult to distinguish from anaplastic carcinoma, particularly on frozen section. Lymphomas generally respond very well to chemotherapy and radiation, with rapid disease regression and remarkable improvement in airway function.

Appropriate evaluation of the extent of the disease should include a complete medical history, physical examination, and imaging studies. Fiberoptic evaluation of the vocal cords is extremely crucial both to evaluate the functionality of the vocal cords, and adequacy of the airway. The glottic chink evaluation is critical in assessment of airway function [7]. It should be recognized that during the fiberoptic laryngoscopy the patient may develop laryngospasm and acute airway distress.

A computed tomography (CT) scan of the chest with contrast is quite helpful to evaluate the extent of disease in the mediastinum and the status of the lungs, and will provide a good demonstration of the airway in a noninvasive manner. Fluorodeoxyglucose positron emission tomography (FDG-PET) scanning also appears helpful for evaluation of the extent of disease in the neck, as well as to identify systemic metastases.

\section{Treatment}

Most patients with anaplastic thyroid carcinoma should be evaluated for treatment by a multidisciplinary tumor board including the pathologist, radiologist, surgeon, radiation oncologist, medical oncologist and endocrinologist. However, ultimate responsibility should lie in the hands of a single individual, as advised by the committee, who will make critical decisions with the family and the patient. A medical oncologist, endocrinologist or surgeon should assume responsibility of the care of the patient depending upon who is best qualified to carry out the treatment plan. All disciplines need to work together and expeditiously for initial evaluation of the disease and planning of treatment. 
The decision regarding resectability of the disease must necessarily be made by the surgeon.

Selected patients with anaplastic thyroid carcinoma with extrathyroidal extension (stage IVB) who show no distant metastases by CT or PET scans and who do not have tumor extending lateral to the carotid arteries are candidates for complete surgical resection [8]. The surgeon is also directly responsible for airway evaluation and managing airway issues both electively and during acute airway distress. It is important to discuss some of the ethical issues with the patient and the family particularly if death is imminent either due to extent of the disease, stage of the anaplastic thyroid carcinoma or severe airway compromise. If the disease can be technically resected surgery should be strongly considered. Although resection of the primary disease may not prolong survival, local control achieved by surgical resection and adjuvant therapy may spare the patient from an agonizing death from airway obstruction. Tumors that involve the trachea, larynx, esophagus or that have extensive soft tissue invasion are generally considered to be inoperable and are best treated with a combination of chemotherapy and radiation therapy. It should be noted that these tumors are not radioiodine sensitive and no effort should be made to administer radioactive iodine ablation either as an initial or postoperative treatment.

\section{Airway issues and anaplastic thyroid carcinoma}

The patient may have a compromised airway, ranging from mild to severe, or airway problems may develop and progress during the course of treatment-particularly during radiotherapy. An acute airway emergency may be precipitated after any manipulation, especially intubation, surgical intervention or even attempted percutaneous endoscopic gastrostomy. Some authors have described the use of endotracheal and endobronchial stents and laser vaporization of intraluminal tumor. These techniques have very limited value for anaplastic thyroid carcinoma and are unlikely to avoid an acute airway catastrophe.

Airway related problems may be caused by extrinsic pressure on the trachea or central compartment of the neck from a large tumor, tracheal invasion by tumor, or paralyzed vocal cords on one or both sides. Paralysis of one cord with impending paralysis of the other side, bilateral vocal cord paralysis, tracheal invasion by the tumor or bleeding inside the trachea most often lead to acute airway distress. Approximately 20-30 \% of patients may present with acute airway problems with mild to moderate stridor. Their airway problems may be accentuated on exertion or minor upper airway infection which may lead to serious compromise of the airway. In the management of anaplastic thyroid carcinoma, airway protection is a critical issue which requires considerable critical thinking and consideration of the quality of life, poor prognosis and limited treatment choices. Conservative treatment such as high humidification and steroids may be of some help temporarily but unlikely to help as the disease progresses. From the time of diagnosis of anaplastic thyroid carcinoma, these issues need to be discussed by the multidisciplinary group as to how to maintain and manage the airway should the patient develop acute airway distress. The issues related to emergency intubation need to be discussed well in advance with the patient and the family. The ethics department of the institution may need to get involved if hard choices are to be made such as "do not resuscitate" (DNR), emergency intubation and ventilatory support. When the patient presents with acute airway problems, it is vitally important to discuss the options with the family so that a rational and thoughtful decision can be made as to how to secure the airway. Clearly, sedating the patient with morphine and allowing the patient to die by natural sequence is another choice; however, most of the patients and the families find this very hard to accept since the entire progression of the disease has usually taken place rapidly over a period of weeks or months. The families often have not had a chance to discuss this among themselves or even with the patient. Once the patient is intubated and unable to speak, input from the patient is unlikely to help make important decisions.

Even though this is a very difficult situation, the airway issues need to be discussed with the patient and the family at the time of initial diagnosis and at the time of impending airway problems or stridor. During the development of ATA guidelines for anaplastic thyroid carcinoma, there was strong input from ethicists. A patient may need intubation if in severe airway distress, cyanotic or the oxygen saturation is dropping rapidly. The patient may need to be intubated on a compassionate basis as there has not been time for a thorough discussion about advance directives including DNR. If the patient presents with acute airway distress and the decision needs to be made about tracheostomy, there are several major issues to be considered:

1. Tracheostomy should be performed under general anesthesia with the airway well secured via intubation. Intubation may be quite difficult because of the large tumor mass and may need a senior experienced anesthesiologist and fiberoptic intubation. The Glidescope (Verathon: Bothell, Washington, USA) may be of great value in these circumstances. Severe orthopnea may require that intubation be performed in a semi-erect position. 
2. Tracheostomy can be quite an undertaking in these patients and finding the trachea may be extremely difficult at the time of tracheostomy. The tumor mass may obstruct exposure of the trachea and the distance between the skin and the trachea may be considerable due to the presence of a large tumor mass. Consideration should be given to cricothyrotomy under these circumstances. The operating surgeon should review the CT scan to evaluate the location of the trachea, displacement of the trachea, and whether there is intratracheal tumor involvement. Generally, cricothyrotomy may be relatively easy as the incision is made above the tumor mass. Sometimes the tumor itself may be in the way of cricothyrotomy and a portion of the tumor mass may be resected for better exposure of the thyroid cartilage and cricoid cartilage. The Harmonic Scapel (Ethicon: Somerville, New Jersey, USA) or LigaSure (Covidien: Mansfield, Massachusetts, USA) devices may be of great help to cut through the tumor for better exposure. A standard tracheostomy tube may be difficult to secure and ensure the tip is beyond the narrowed region of the trachea. A long tracheostomy tube of $110 \mathrm{~mm}$ is generally a better choice. In an emergency situation an endotracheal tube can be placed through the cricothyrotomy opening. The tube should be well secured to avoid any dislodgement. Tracheal secretions and cleaning of the tracheostomy tube may be quite difficult in these individuals. Patients may require repeated bronchoscopies to clean the tracheostomy tube and distal trachea.

Generally, prophylactic tracheostomy should be avoided as the quality of life suffers considerably in these individuals, and is best avoided until there is an impending airway problem. We tend to avoid prophylactic tracheostomy in patients undergoing radiation therapy. During the course of radiation therapy, patients should be closely monitored and may need hospitalization to stabilize the airway with humidification, steroids, etc.

3. Technical aspects of the tracheostomy or cricothyrotomy may be quite difficult unless the surgeon is well experienced with anaplastic thyroid carcinoma. Unfortunately, even after tracheostomy, the airway problems may continue especially with the common occurrence of tumor growing through the tracheostomy opening and skin incision. Bleeding through the tracheostomy due to tumor is also a perplexing problem. The tumor may fungate through the incision and may obstruct the airway again at time where there are hardly any choices remaining to maintain a patent airway. Shaha [9] described his experience of handling the airway in 30 patients with anaplastic thyroid carcinoma. Even though there is no strong consensus, a patient may need a surgical airway if there is an impending acute airway catastrophe. Other authors have had similar unfortunate experiences with tracheostomy and anaplastic thyroid carcinoma in which the quality of life deteriorates rapidly with obstruction and bleeding caused by intratracheal tumor [10]. It can be quite appropriate to ask for help from senior colleagues or surgeons who have more experience with managing the airway in anaplastic thyroid carcinoma. Once performed, the tracheostomy should be well secured with retention stitches and a tape around the neck. It is important to make sure that the tip of the tracheostomy tube is well below the obstruction in the trachea. Occasionally, the intraluminal tumor can cause bleeding or continued airway difficulty.

After tracheostomy, the majority of patients end up staying an extended period of time in the hospital or are transferred to hospice care. Outpatient radiation therapy is difficult in terms of logistics and quality of life and the patient may need hospitalization. The quality of life may deteriorate considerably in some patients. Families often find it very hard to make any definitive decisions and will need several consultation sessions by various disciplines including nursing, ethics and social work. The ATA anaplastic guidelines related to airway are listed here [6]. Recommendations 25-28:

\section{RECOMMENDATION 25}

Patients with ATC may require tracheostomy to avoid asphyxia. It should be done in an operating room setting, unless acute airway distress demands immediate action.

Strength of Recommendation: Strong

Quality of evidence: Low

RECOMMENDATION 26

The patient's airway should be closely monitored in the recovery room during the postoperative period and throughout radiation therapy. Elective tracheostomy is best avoided unless there are acute airway issues.

Strength of Recommendation: Strong

Quality of evidence: Low

RECOMMENDATION 27

Tracheostomy may be temporarily beneficial in patients with impending airway loss.

Strength of Recommendation: Strong

Quality of evidence: Low

\section{RECOMMENDATION 28}

Tracheostomy or stent placement is best avoided unless there is impending airway compromise.

Strength of Recommendation: Strong

Quality of evidence: Low 
Recommendations 17 and 18 are included regarding treatment philosophy [6].

\section{RECOMMENDATION 17}

In consultation with a multidisciplinary team, a candid meeting with the patient should be scheduled in which there is full disclosure of the potential risks and benefits of various treatment options, including how such options will impact the patient's life. Treatment options discussed should include palliative care. Patient preferences should guide clinical management.

Strength of Recommendation: Strong

Quality of evidence: Low

\section{RECOMMENDATION 18}

Patients should be encouraged to draft an advance directive in which they name a surrogate decision maker and list code status and other end-of-life preferences. Consider, in some cases, using "allow natural death" (AND) over DNR, which may be better understood by patients and families as an order that limits inappropriate aggressive care. Circumstances in which suspension of DNR or AND may occur must be discussed with the patient.

Strength of Recommendation: Strong

Quality of evidence: Low

\section{Conclusion}

Anaplastic thyroid carcinoma continues to be one of the most challenging and distressing human cancers with an almost uniformly lethal outcome. Immunohistochemical and detailed histopathological evaluation help to distinguish the aggressiveness of anaplastic thyroid carcinoma. The treatment regimen generally involves a combination of chemotherapy and radiation therapy. However, it is important to determine whether the disease is surgically resectable. The majority of patients will develop nodal and distant metastases. Airway issues continue to be a major concern, and securing a safe airway may be extremely difficult. The role of elective tracheostomy generates considerable debate. Tracheotomy may be considered for impending acute airway distress which may prolong life for a short period; it may also prolong suffering. The ethical issues in the management of anaplastic thyroid carcinoma need to be discussed with the patient and the family at length.

\section{References}

1. Smallridge RC, Copland JA (2010) Anaplastic thyroid carcinoma: pathogenesis and emerging therapies. Clin Oncol (R Coll Radiol) 22:486-497

2. Smallridge RC (2012) Approach to the patient with anaplastic thyroid carcinoma. J Clin Endocrinol Metab 97:2566-2572

3. Kim JH, Leeper RD (1983) Treatment of anaplastic giant and spindle cell carcinoma of the thyroid gland with combination Adriamycin and radiation therapy. A new approach. Cancer 52:954-957

4. Sherman EJ, Lim SH, Ho AL et al (2011) Concurrent doxorubicin and radiotherapy for anaplastic thyroid cancer: a critical reevaluation including uniform pathologic review. Radiother Oncol 101:425-430

5. Are C, Shaha AR (2006) Anaplastic thyroid carcinoma: biology, pathogenesis, prognostic factors and treatment approaches. Ann Surg Oncol 13:435-464

6. Smallridge RC, Ain KB, Asa SL et al (2012) American Thyroid Association guidelines for management of patients with anaplastic thyroid cancer. Thyroid 22:1104-1139

7. Chandran S, Hanna J, Lurie D, Sataloff RT (2011) Differences between flexible and rigid endoscopy in assessing the posterior glottis chink. J Voice 25:591-595

8. Brown RF, Ducic Y (2013) Aggressive surgical resection of anaplastic thyroid carcinoma may provide long-term survival in selected patients. Otolaryngol Head Neck Surg 148:564-571

9. Shaha AR (2008) Airway management in anaplastic thyroid carcinoma. Laryngoscope 118:1195-1198

10. Hölting T, Meybier H, Buhr H (1989) Problems of tracheotomy in locally invasive anaplastic thyroid cancer. Langenbecks Arch Chir 374:72-76 [in German] 